\title{
Infrared imaging Fourier-transform spectrometer used for standoff gas detection
}

\author{
M. Kastek, T. Piątkowski \& H. Polakowski \\ Institute of Optoelectronics, Military University of Technology, Poland
}

\begin{abstract}
The article presents the detection of gases using an infrared imaging Fouriertransform spectrometer (IFTS). The Telops Company has developed the IFTS instrument HyperCam, which is offered as short or long wave infrared device. The principle of HyperCam operation and methodology of gases detection has been shown in the paper, as well as theoretical evaluation of gases detection possibility. The calculation of an optical path between an IFTS device, cloud of gases and background has been also discussed. The variation of a signal reaching the IFTS caused by the presence of a gas has been calculated and compared with the reference signal obtained without the presence of a gas in IFTS's field of view. Verification of theoretical result has been made by laboratory measurements. Some results of the detection of various types of gases have also been included in the paper.
\end{abstract}

Keywords: gas detection, hyperspectral detection, imaging Fourier-transform spectrometer, stand-off detection.

\section{Introduction}

Fourier-transform spectrometers (FTS) are renowned instruments, particularly well-suited to remotely provide excellent estimates of quantitative data. Many authors have presented how they are using conventional (non-imaging) FTS to perform quantification of distant gas emissions. Amongst others, we should mention the important contributions made by Harig and Matz [1] and Hang et al. [2]. His group performed many measurement campaigns for which they obtained excellent results by ensuring proper modeling of the scene and by paying attention to understand (and to take into account) the instrument signature. Other groups developed similar approaches however limiting their study to optically 
thin plumes $[3,4]$ to dedicated instruments performing optical subtraction $[5,6]$, or introducing Bayesian algorithms to maximize the use of a priori information [7]. On the other hand, detection and quantification activities with an imaging Fourier-transform spectrometer (IFTS) have been first presented by Spisz et al. [8].

The present paper deals with the remote gas identification and quantification from turbulent stack plumes with an IFTS. It presents first the modeling that is required in order to get an appropriate understanding of both the scene and the instrument. Next it covers the methodology of the developed quantification approach. Finally, results are presented to demonstrate the capabilities and the performances of the remote gas quantification by using hyperspectral data obtained from the Telops HyperCam. The latter is described in much detail in prior references [9-12].

\section{Instrumentation}

The infrared imaging Fourier-transform spectrometer (IFTS) - HyperCam - used on experiment was built by Telops, Inc. This IFTS has 320 x 256 pixel Mercury Cadmium Telluride (MCT) focal plane arrays (FPA) with a $6^{\circ}$ x $5^{\circ} \mathrm{FOV}$. The FPAs are Sterling cooled to provide good noise figures in a field ready package. Spectral information is obtained using a technique called Fourier Transform Infrared Radiometry (FTIR).

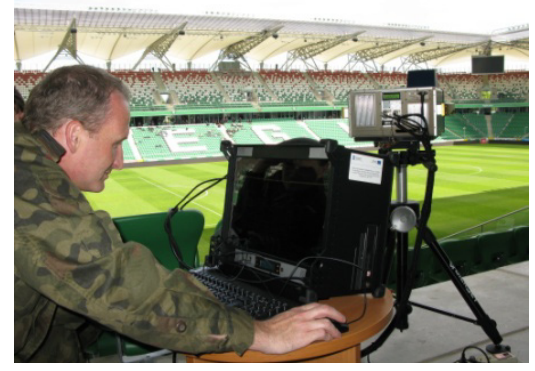

Figure 1: HyperCam sensor during the measurement.

FTIR is a classical interference based technique applied to gas spectroscopy that uses a Michelson interferometer to mix an incoming signal with itself at several different discrete time delays. The resulting time domain waveform, called an interferogram, is related to the power spectrum of the scene through the Fourier transform. An interferogram for each pixel in an image is created by imaging the output of the interferometer onto a focal plane array and collecting data at each discrete time delay. Advantages of using an FTIR sensor over a grating based (filter) system include higher resolution for equal cost and the absence of misalignment of different color images due to platform motion. However, FTIR does have the disadvantage of producing a slower frame rate than filter based systems because twice as many points are taken for the same 
number of spectral points. For atmospheric tracking of gases however, the HyperCam sensor has sufficient frame rate $[8,11]$. Data for this collection can be taken at $0.25-150 \mathrm{~cm}^{-1}$ spectral resolution between $830 \mathrm{~cm}^{-1}(12 \mu \mathrm{m})$ and $1290 \mathrm{~cm}^{-1}(7.75 \mu \mathrm{m})$ at a frame rate of $0.2 \mathrm{~Hz}$. In addition to the infrared data, visible imagery was taken using a firewire camera that is boresighted to the IR sensor. Fig. 1 shows a picture of a HyperCam sensor during the measurement. The sensor is controlled by field computer and data is stored on a RAID drive to guarantee integrity of data [12]. The package of software Reveal is prepared for control and registration of data from HyperCam, and for calibration of raw data, and for analysis of these results. Fig. 2 presents the windows of software Reveal Pro for control parameters of HyperCam and registered data. Fig. 3 shows the part of software for data analysis.

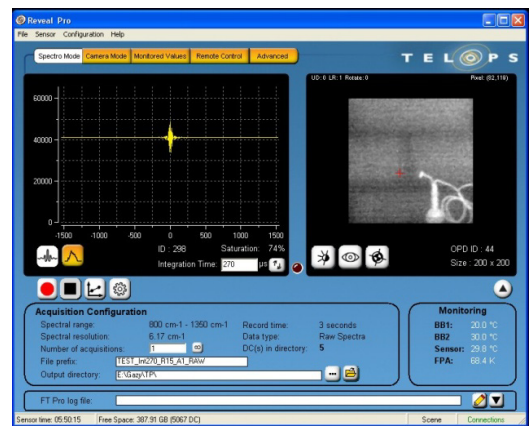

Figure 2: $\quad$ Software Reveal Pro for control HyperCam sensor.

(a)

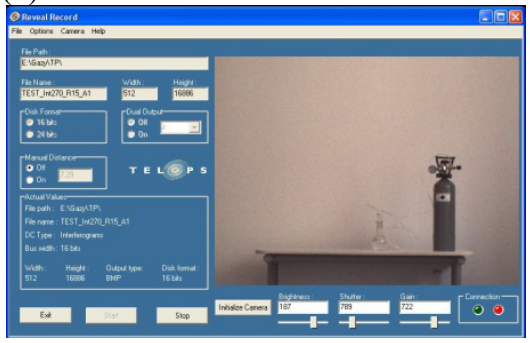

(b)

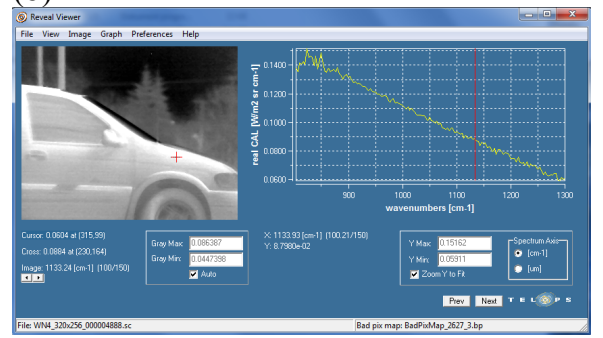

Figure 3: $\quad$ Software for data analysis: registration visible picture (a), analysis of spectral data (b).

\section{Model of process detection}

The physical problem of interest in the present paper concerns imaging Fouriertransform spectrometers taking measurements from a ground-based platform. Nevertheless, the approach described herein directly applies, with some adjustments, to airborne-based measurements. In the typical setup the instrument 
is looking towards a stack releasing gases. The scene, from the instrument pointof-view, is implicitly inhomogeneous. Vertical variations of temperature and gas concentrations are necessarily present both in the released gases and in the atmospheric background. From the small instrument instantaneous field-of-view (IFOV) perspective, the instrument does see a small solid angle in a given direction, thus sensing a narrow pencil of air mass. Within the illustrated disposition, any line of pixels therefore provides a complete section of the plume. Modelling the experimental conditions illustrated in Fig. 3 may be done by considering fundamentally two distinct aspects. The first deals with the whole contribution of the released gases through the atmosphere. And the second is devoted to understanding how the instrument itself does add its own signature into the measurement $[11,12]$.

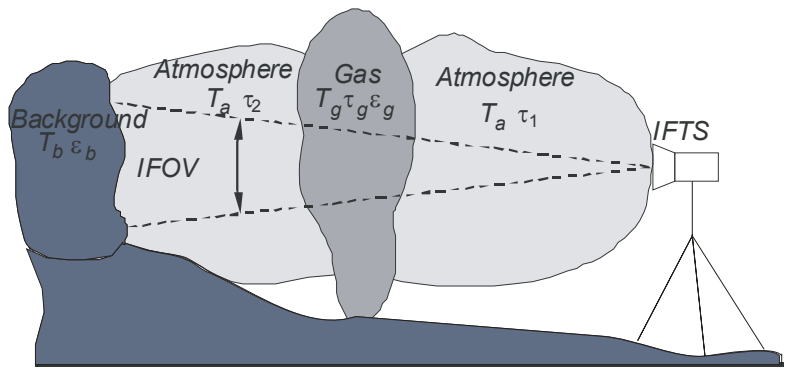

Figure 4: Scheme of a measuring process and notations accepted for gas analysis in atmosphere.

In order to detect the gas plume, it is essential to begin with an underlying physics-based model for the hyperspectral data. This allows us to mathematically describe the radiance, defined as the flux per unit projected area per unit solid angle, incident at the sensor. This at-sensor radiance, $L(\lambda)$ is a summation of the contributions of numerous terms including both reflected solar and emitted thermal effects. In the LWIR, emissive terms dominate and reflective effects are generally considered negligible [12].

For ground-based observation of a gaseous plume, the at-sensor radiance can be described as:

$$
\mathrm{L}(\lambda)=\mathrm{L}_{\mathrm{g}}(\lambda)+\mathrm{L}_{\mathrm{b}+\mathrm{g}}(\lambda)+\mathrm{L}_{\mathrm{a}}(\lambda)
$$

where $L_{g}(\lambda)$ is the self-emitted radiance from the gas plume at wavenumber $\lambda$ $\left(\mathrm{cm}^{-1}\right), L_{b+g}(\lambda)$ is the self-emitted radiance from the background transmitted through the plume, and $L_{a}(\lambda)$ is the scattered self-emitted atmospheric effects (also known as upwelled radiance). The self-emitted radiance from the gaseous plume can be expanded such that

$$
\mathrm{L}_{\mathrm{g}}(\lambda)=\tau_{1}(\lambda) \varepsilon_{\mathrm{g}}(\lambda) \mathrm{B}\left(\lambda, \mathrm{T}_{\mathrm{g}}\right)
$$

where $\tau_{l}(\lambda)$ is the atmospheric transmittance from the gas plume to the sensor, $\varepsilon_{g}(\lambda)$ is the effective emissivity of the plume material, $T_{g}$ is the temperature of the gas plume in Kelvin, and 


$$
\mathrm{B}(\lambda, \mathrm{T})=\frac{2 \pi \mathrm{hc} \mathrm{c}^{2} \lambda^{3}}{\mathrm{e}^{\left(\frac{\mathrm{hc} \lambda}{\mathrm{kT}}\right)}-1}
$$

is Planck's blackbody curve where $k$ is Boltzmann's constant, $c$ is the speed of light, $h$ is Planck's constant, and $\lambda$ is wavenumber $\left(\mathrm{cm}^{-1}\right)$. Therefore the radiance seen at the sensor due to the gas plume is a function of the gas plume's emissivity, its temperature, and the atmosphere between it and the sensor. For the background, the radiance seen at the sensor is more complicated. This radiance is defined as

$$
\mathrm{L}_{\mathrm{b}+\mathrm{g}}(\lambda)=\tau_{1}(\lambda) \tau_{\mathrm{g}}(\lambda) \tau_{2}(\lambda) \varepsilon_{\mathrm{b}}(\lambda) \mathrm{B}\left(\lambda, \mathrm{T}_{\mathrm{b}}\right)
$$

where $\tau_{g}(\lambda)$ is the transmittance through the gas plume, $\tau_{2}(\lambda)$ is the atmospheric transmittance between the background and the gas plume, and $\varepsilon_{b}(\lambda)$ is the emissivity of the background material. In this case, the photons from the background travel through the atmosphere to the gas plume. The photons then travel through the gas plume which has its own transmittance function. The photons that make it through the gas plume travel the final distance to the sensor. Equation (4) can be simplified by assuming the gas plume is optically thin. Under this assumption, the Beer-Lambert Law states the gas plume transmittance is a function of the gas plumes effective emissivity such that

$$
\tau_{\mathrm{g}}(\lambda)=1-\varepsilon_{\mathrm{b}}(\lambda)
$$

From equations (2), (4), and (5), (1) and using some simple algebra, (6) can be rewritten such that

$$
\begin{gathered}
\mathrm{L}(\lambda)=\tau_{1}(\lambda) \tau_{\mathrm{g}}(\lambda)\left[\mathrm{B}\left(\lambda, \mathrm{T}_{\mathrm{g}}\right)-\tau_{2}(\lambda) \varepsilon_{\mathrm{b}}(\lambda) \mathrm{B}\left(\lambda, \mathrm{T}_{\mathrm{b}}\right)\right]+ \\
\tau_{1}(\lambda) \tau_{2}(\lambda) \varepsilon_{\mathrm{b}}(\lambda) \mathrm{B}\left(\lambda, \mathrm{T}_{\mathrm{b}}\right)+\mathrm{L}_{\mathrm{s}}(\lambda)
\end{gathered}
$$

To solve (6), we have to know something about the atmospheric transmission, the temperature of the background, the temperature of the gas plume, the emissivity signature of the background, the scattering (or up welled radiance) term, and the emissivity signature of the plume.

Unfortunately, we only know the emissivity signature of the gas plume and the ambient air temperature; therefore, we must make some simplifying assumptions to use (6). The first assumption we make is that the atmospheric terms are negligible and/or are the same for all terms because all objects are relatively close to the camera. For the same reason, the second assumption we can make is that the up welled radiance term is also minimal and may be dropped. These assumptions lead to the following simplified model:

$$
\mathrm{L}(\lambda) \approx \tau_{\mathrm{g}}(\lambda)\left[\mathrm{B}\left(\lambda, \mathrm{T}_{\mathrm{g}}\right)-\varepsilon_{\mathrm{b}}(\lambda) \mathrm{B}\left(\lambda, \mathrm{T}_{\mathrm{b}}\right)\right]+\varepsilon_{\mathrm{b}}(\lambda) \mathrm{B}\left(\lambda, \mathrm{T}_{\mathrm{b}}\right)
$$

Equation (7) can be expressed in terms of apparent emissivity if an estimate of $B\left(\lambda, T_{b}\right)$ can be calculated. To do this, the temperature at each pixel can be calculated such that

$$
\mathrm{T}=\frac{\mathrm{hc} \lambda}{\mathrm{k} \log \left(\frac{2 \mathrm{hc^{2 } \lambda ^ { 3 }}}{\mathrm{L}(\lambda)}+1\right)}
$$

where $L(\lambda)$ is the radiance signature for the pixel under test, $k$ is Boltzmann's 
constant, $c$ is the speed of light, $h$ is Planck's constant, and $\lambda$ is wavenumber $\left(\mathrm{cm}^{-1}\right)$. Using (3) and (8), an estimate for the temperature and Planck's blackbody curve can be created for every pixel in the scene. Each pixel can be divided by its estimated blackbody curve to obtain an estimate of its apparent emissivity:

$$
\varepsilon(\lambda) \approx \varepsilon_{\mathrm{g}}(\lambda)\left[\frac{\mathrm{B}\left(\lambda, \mathrm{T}_{\mathrm{g}}\right)}{\mathrm{B}\left(\lambda, \mathrm{T}_{\mathrm{b}}\right)}-\varepsilon_{\mathrm{b}}(\lambda)\right]+\varepsilon_{\mathrm{b}}(\lambda)
$$

Another assumption we can make is that the factor multiplying plume emissivity can be roughly approximated as a function of the temperatures between the plume and the background:

$$
\left[\frac{\mathrm{B}\left(\lambda, \mathrm{T}_{\mathrm{g}}\right)}{\mathrm{B}\left(\lambda, \mathrm{T}_{\mathrm{b}}\right)}-\varepsilon_{\mathrm{b}}(\lambda)\right] \approx \mathrm{f}\left(\mathrm{T}_{\mathrm{g}}-\mathrm{T}_{\mathrm{b}}\right)
$$

These assumptions of course could be removed and more robust atmospheric modeling and compensation implemented.

However, we have found that, for the relatively close stand-off distances considered, the current simplified model yields good results. Thus using the assumption in (10), a new apparent emissivity model is described by

$$
\varepsilon(\lambda) \approx \varepsilon_{\mathrm{g}}(\lambda) \mathrm{f}\left(\mathrm{T}_{\mathrm{g}}-\mathrm{T}_{\mathrm{b}}\right)+\varepsilon_{\mathrm{b}}(\lambda)
$$

Equation (11) suggests the background can be modeled by one emissivity; however, the background is typically comprised of multiple materials each with their own emissivity. In such a case, the apparent emissivity of the background is

$$
\varepsilon_{\mathrm{b}}(\lambda)=\sum_{\mathrm{j}=1}^{\mathrm{Q}} \beta_{\mathrm{j}} \varepsilon_{\mathrm{j}}(\lambda)
$$

where $Q$ is the number of unique background emissivity signatures, $\varepsilon_{j}$ is the emittance for the $\mathrm{j}^{\text {th }}$ background signature and $\beta_{j}$ is the amount of $\mathrm{j}^{\text {th }}$ background material. Using (12), the final apparent emissivity model is

$$
\varepsilon(\lambda) \approx \varepsilon_{\mathrm{g}}(\lambda) \beta_{0}+\sum_{\mathrm{j}=1}^{\mathrm{Q}} \beta_{\mathrm{j}} \varepsilon_{\mathrm{j}}(\lambda)
$$

where

$$
\beta_{0}=\mathrm{f}\left(\mathrm{T}_{\mathrm{g}}-\mathrm{T}_{\mathrm{b}}\right)
$$

Therefore, each pixel in the scene is processed in the following manner. First, the apparent temperature is estimated using (8). From this estimate, the Planck's blackbody radiation curve is generated according to (3). The pixel is divided by its blackbody radiation estimate to obtain (13). It is this equation can be used at the detection algorithm of gases. The algorithm of automation gases detection was under development and tests $[10,12]$. The influence of emissivity coefficient on measurement results can also be compensated by adopting several methods from pyrometric non-contact temperature measurements. There are many known methods [13-16] for such compensation and an algorithm taking into account real emissivity values should be included in automatic gas detection procedures.

In the long infrared wave spectral range (LWIR), for chemicals in the gaseous form, scattering processes are not expected to contribute, so they are neglected. 
Note that this assumption would not be valid when aerosols are present. In general the spectral absorption coefficients may be dependent upon light polarization. However atmospheric absorption (and emission) being due to molecules, polarization dependence is not expected. Accordingly, the present description does not include polarization effects.

The absorption coefficient is made of a linear combination over the gas compounds of the atmosphere (linear mixture model). These coefficients are obtained from gas reference databases of infrared signatures (often in absolute absorbance's) as a function of the gas temperature (e.g. HITRAN molecular spectroscopic database [17] and Pacific Northwest National Laboratory (PNNL) Spectral Library [18]).

The transmission of the cloud gas $\tau_{g}$ is computed from the spectral properties of the chemical species using Beers' Law:

$$
\tau_{m}(\lambda)=\exp \left[-\sum k_{i}(\lambda) C_{i} d\right],
$$

where $C_{i}$ is the average concentration of the chemical compound over the path length $d$ and $k_{i}(\lambda)$ is the wavelength-dependent absorption coefficient.

The sum over index $i$ in Eq. (15) is over all spectrally relevant chemical species. For the computer simulation of transmission atmosphere with gas (e.g. methane) was used the PC MODWIN 3 v.1.0 computer program for simulation of transmission of atmosphere $[19,20]$.

IFTS technology can be used for the gas quantification but of course the methodology of this is now under the testing. This kind of algorithm used some of filtering methods and method comparison results of measurement with model results [21]. A measurement session was performed in the laboratory and in the open-space conditions to practically verify the possibility of applying IFTS-type spectroradiometer for gas detection. The HyperCam LWIR device was used for the experiment and four different gases were observed. During the laboratory experiments the ambient temperature and humidity were monitored and during open-space tests the wind speed and direction and atmospheric pressure were additionally recorded.

\section{Laboratory tests}

The laboratory tests were performed in order to test the effectiveness of imaging Fourier-transform spectroradiometer as a tool for the measurement of spectral characteristics of single gases or gas mixtures. The real photo of the laboratory test stand used during experiments is depicted in Fig. 5.

The measurements on a test stand presented in Fig. 5. were performed in order to determine the minimal gas concentration which can be detected by HyperCam LWIR spectroradiometer. The flow from gas cylinder was controlled by a precise gas pressure regulator and large-area blackbody was used to determine the value of thermal contrast between gas cloud and background. The applied blackbody has a radiative area of $11 \times 11$ inch and its temperature difference $\Delta \mathrm{T}$ can be set with respect to ambient or temperature value measured 


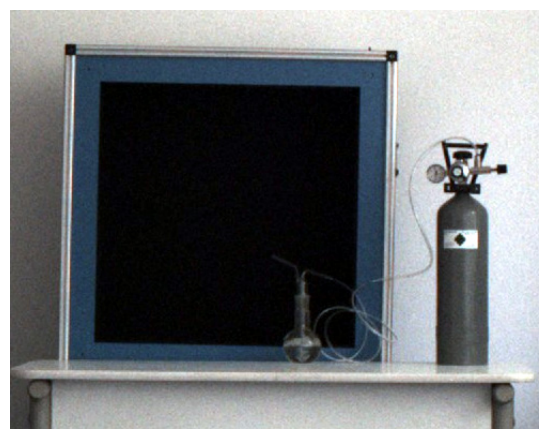

Figure 5: The laboratory test stand.

by an external sensor. In our case, this external sensor was placed at the output of gas nozzle thus the thermal contrast between gas cloud and background (blackbody area) could be precisely set.

The following gases were used during the measurements: $\mathrm{NO}_{2}, \mathrm{CO}_{2}$ and propane-butane mixture, all of those having absorption lines in far infrared spectral range. Theoretical absorption spectra for $\mathrm{CO}_{2}$ and $\mathrm{NO}_{2}$ gases calculated by HITRAN software are presented in Fig. 6. Those characteristics were calculated for the same environmental conditions as recorded during laboratory tests, i.e. ambient temperature 294.20 K, and using Middle Latitude Summer atmosphere model from commonly available database of atmospheric data from Modtran software. It can be seen from Fig. 6 plots that calculated spectra fit well inside the measurement range of HyperCam LWIR spectroradiometer and it can be expected that both gases should be detectable using this device.

(a)

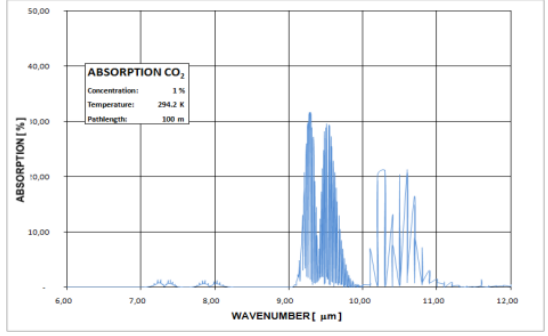

(b)

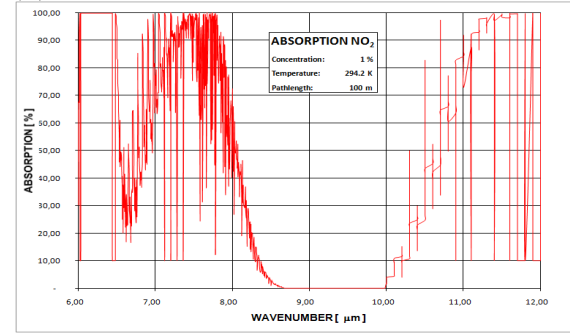

Figure 6: The absorption $\mathrm{CO}_{2}$ (concentration 1\%) (a), $\mathrm{NO}_{2}$ (concentration $1 \%$ ) (b).

The applied gas pressure regulator provided the controlled gas flow, during the measurement session the flow was set at $5 \mathrm{mg} / \mathrm{s}$ which corresponds to gas concentration of $1 \%$ in the measurement zone observed by HyperCam LWIR. Spectra characteristics of gases were measured for the following values of thermal contrast: $\Delta \mathrm{T}=1 \mathrm{~K}, \Delta \mathrm{T}=2 \mathrm{~K}, \Delta \mathrm{T}=3 \mathrm{~K}$. In order to obtain best possible accuracy the spectral resolution was set at $0,75 \mathrm{~cm}^{-1}$ whereas the frame rate was $0.3 \mathrm{~Hz}$ and it resulted from chosen resolution of sub-frame windowing (limited area of an array used during measurements which also reduced the field of view). 
Each measurement was repeated twice in order to avoid accidental errors. The results obtained from HyperCam LWIR spectroradiometer gave finally the spectral characteristics of selected gases for different measurement conditions. In Fig. 7 results of experiments on measurement of characteristics of $\mathrm{CO}_{2}$ and $\mathrm{NO}_{2}$ are presented.

(a)

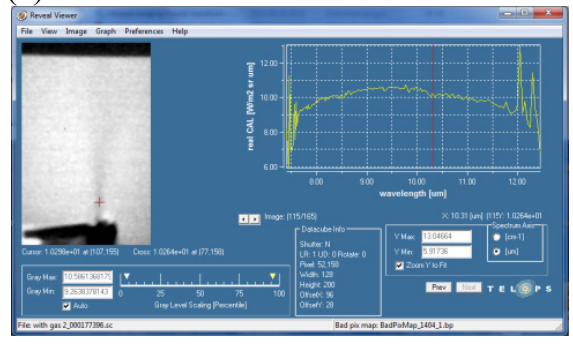

(b)

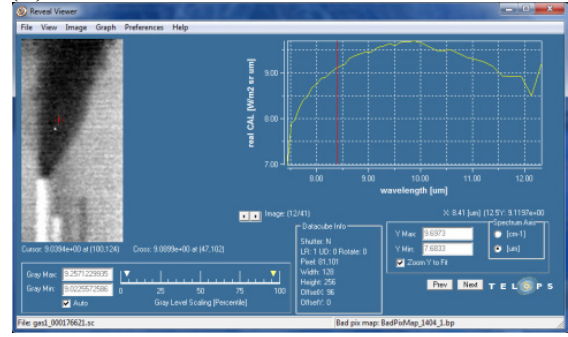

Figure 7: The results of measure $\mathrm{CO}_{2}$ (concentration 1\%) (a), $\mathrm{NO}_{2}$ (concentration 1\%) (b) on laboratory tests.

\section{$5 \quad$ Field experiments}

During the field tests the following gases was measured: $\mathrm{CO}_{2}$, propane-butane mixture and freon 134 (CH2FCF3 tetrafluoroethane). In each case gas concentrations were tested in order to verify the efficiency of gas measurements in the open space by HyperCam LWIR.

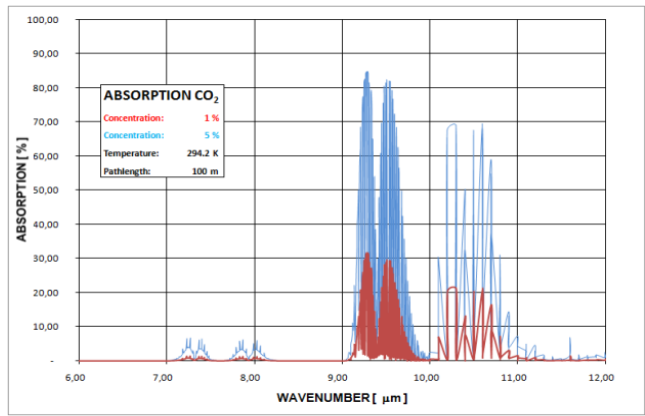

Figure 8: The theoretical absorption $\mathrm{CO}_{2}$ for different concentration $1 \%$ and $5 \%$.

Theoretical absorption spectra for two concentrations of $\mathrm{CO}_{2}(1 \%$ and $5 \%)$ were calculated by HITRAN software are presented in Fig. 8. As expected, higher gas concentration significantly increases the absorption and broadens its spectral range. During the experiment measured absorption characteristics of $\mathrm{CO}_{2}$ for two concentrations were measured too. The results of this measurement gives possibility to detect gases on natural environments used the HyperCam LWIR. During the field measurements the weather conditions were monitored by 
(a)

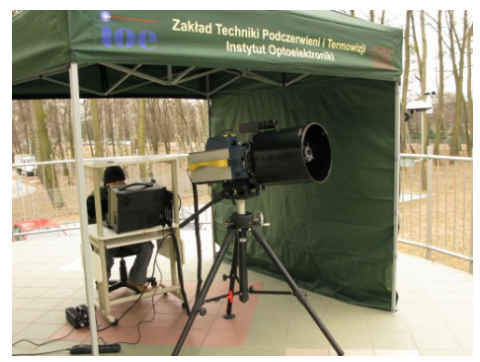

(b)

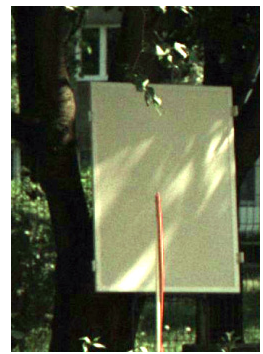

Figure 9: The measure during field test on MUT area: spectroradiometer HyperCam (a), pipe with gas and plate as a background (b).

an automatic weather station. The measurement set-up with HyperCam LWIR spectroradiometer during field tests is shown in Fig. 9.

As already mentioned, during the field tests the freon 134 gas was used. This composition it exhibits absorption bands in long wave infrared range, thus it can be detected using HyperCam LWIR. The recordings were made at two distances: $60 \mathrm{~m}$ and $10 \mathrm{~m}$, for two gas concentrations of $3 \%$ and $6 \%$. The results are presented in Figs. 10 and 11. Additionally, some Matlab-based post-processing results are also presented.

(a)

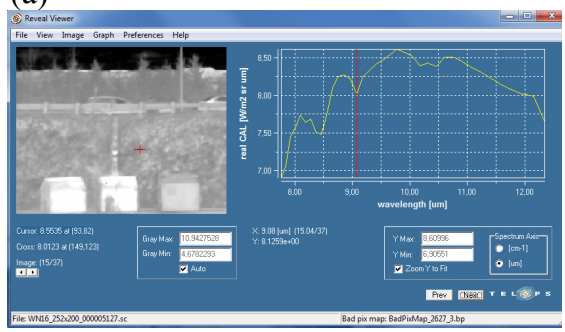

(b)

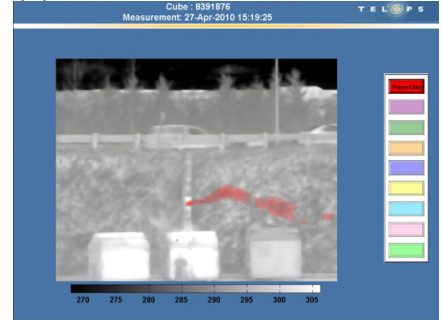

Figure 10: The results of measure of freon 134 (concentration 3\%) distance $60 \mathrm{~m}$ (a), freon 134 results of analysis in Matlab (b).

(a)

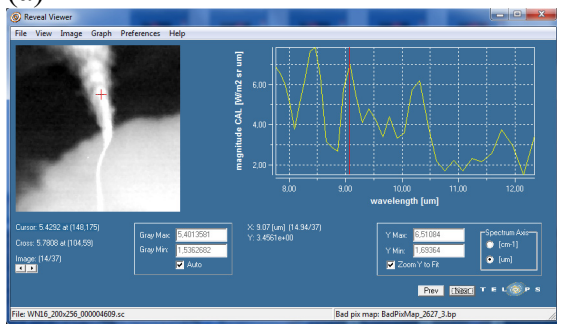

(b)

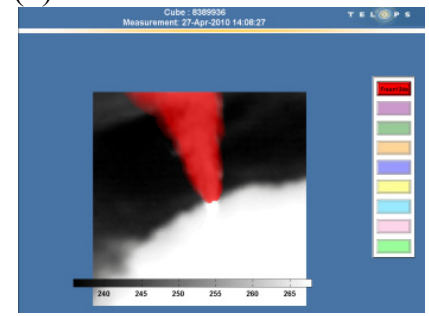

Figure 11: The results of measure of freon 134 (concentration 6\%) distance $10 \mathrm{~m}$ (a), freon 134 results of analysis in Matlab (b). 


\section{Conclusion}

After the analysis of result laboratory tests and tests field efficiency of the imaging Fourier-transform spectrometer technology was confirmed. During the experiment measurement of characteristics of gases were made with different conditions (different concentration) and during different environments conditions. For the over side the results of tests confirmed possibility to use this kind of spectroradiometers to automatically detection of gases, after the development method and software for it.

The results presented in the paper are supported by realization of the Project is co-financed by the European Regional Development Fund within the framework of the 2. priority axis of the Innovative Economy Operational Programme, 2007-2013, submeasure 2.1. "The development of centres with high research potential” Contract no. POIG.02.01.00-14-095/09.

\section{References}

[1] Harig R., Matz G., "Toxic Cloud Imaging by Infrared Spectrometry: A Scanning FTIR System for Identification and Visualization", Field Analytical Chemistry and Technology, 5(1-2), 75-90 (2001).

[2] Harig R., Matz G., Rusch P., "Scanning Infrared Remote Sensing System for Identification, Visualization, and Quantification of Airborne Pollutants", Proc. of SPIE 4574, 83-94 (2002).

[3] Griffin M. K., Kerekes J. P., Farrar K. E., Burke H.-H. K., "Characterization of Gaseous Effluents from Modeling of LWIR Hyperspectral Measurements", Proc. of SPIE 4381, 360-369 (2001).

[4] Burr T., Hengartner N., "Overview of Physical Models and Statistical Approaches for Weak Gaseous Plume Detection using Passive Infrared Hyperspectral Imagery", Sensors, 6(12), 1721-1750 (2006).

[5] Lachance R. L., Thériault J.-M., Lafond C., Villemaire A. J., "Gaseous emanation detection algorithm using a Fourier transform interferometer operating in differential mode", Proc. of SPIE 3383, 124 (1998).

[6] Thériault J.-M., "Passive standoff detection of chemical vapors by differential FTIR radiometry", Technical Report Defence Research Establishment Valcartier (DREV) TR-2000-156 (2001).

[7] Heasler P., Posse C., Hylden J., Anderson K., "Nonlinear Bayesian Algorithms for Gas Plume Detection and Estimation from Hyper-spectral Thermal Image Data”, Sensors 7, 905-920 (2007).

[8] Spisz T. S., Murphy P. K., Carter C .C., Carr A. K., Vallières A., Chamberland M., "Field test results of standoff chemical detection using the FIRST", Proc. of SPIE 6554, 655408 (2007).

[9] Farley V., Chamberland M., Lagueux P., Vallières A., Villemaire A., Giroux J., "Chemical agent detection and identification with a hyperspectral imaging infrared sensor", Proc. of SPIE 6661, 66610L (2007). 
[10] Vallières A., Villemaire A., Chamberland M., Belhumeur L., Farley V., Giroux J., Legault J.-F., “Algorithms for chemical detection, identification and quantification for thermal hyperspectral imagers", Proc. of SPIE 5995, 59950G (2005).

[11] Chamberland M., Belzile C., Farley V., Legault J.-F., Schwantes K., "Advancements in field-portable imaging radiometric spectrometer technology for chemical detection", Proc. of SPIE 5416, 63-72 (2004).

[12] Farley V., Belzile C.; Chamberland M., Legault J.-F., Schwantes K., "Development and testing of a hyper-spectral imaging instrument for field spectroscopy", Proc. of SPIE 5546, 29-36 (2004).

[13] Madura H., Kastek M., Piątkowski T., "Automatic compensation of emissivity in three-wavelength pyrometers", Infrared Physics \& Technology, Volume 51, Issue 1, July 2007, Pages 1-8.

[14] Madura H., Kastek M., Sosnowski T., Orżąnowski T., "Pyrometric method of temperature measurement with compensation for solar radiation", Metrology and Measurement Systems, Volume 17, Issue 1, 2010.

[15] Bielecki, Z., Chrzanowski, K., Matyszkiel, R., Piạtkowski, T., Szulim, M., "Infrared pyrometer for temperature measurement of objects of both wavelength- and time-dependent emissivity", Optica Applicata, Volume 29, Issue 3, 1999, Pages 284-292.

[16] Madura, H. , Piątkowski, T., Powiada, E., "Multispectral precise pyrometer for measurement of seawater surface temperature", Infrared Physics \& Technology, Volume 46, Issue 1-2 SPEC. ISS., December 2004, Pages 6973.

[17] Champion J.-P., Chance K., Coudert L. H., et al., "The HITRAN 2008 molecular spectroscopic database", Journal of Quantitative Spectroscopy and Radiative Transfer 110, 533-572 (2009).

[18] Sharpe S. W., Johnson T. J., Sams R. L., Chu P. M., Rhoderick G. C., Johnson P. A., "Gas-Phase Databases for Quantitative Infrared Spectroscopy”, Applied Spectroscopy 58(12), 1452-1461 (2004).

[19] Kastek M., Sosnowski T., Orżanowski T., Kopczyński K., Kwaśny M., "Multispectral gas detection method", WIT Transactions on Ecology and the Environment Volume 123, pp. 227-236 (2009).

[20] Włodarski M., Kopczyński K., Kaliszewski M., Kwaśny M., MularczykOliwa M., Kastek M., "Application of advanced optical methods for classification of air contaminants", WIT Transactions on Ecology and the Environment Volume 123, pp.237-247 (2009).

[21] Tremblay P., Savary S., Rolland M., Villemaire A., Chamberland M., Farley V. et al., "Standoff gas identification and quantification from turbulent stack plumes with an imaging Fourier-transform spectrometer", Proc. of SPIE Vol. 7673, 76730H (2010). 